Vol. 82 (1992)

\title{
X-RAY STANDING WAVE TECHNIQUE AS A TOOL IN THE STUDY OF THE IMPERFECT CRYSTALS
}

\author{
J. Pelka and J. Auleytner \\ Institute of Physics, Polish Academy of Sciences \\ Al. Lotników 32/46, 02-668 Warsaw, Poland
}

\begin{abstract}
The application of the X-ray standing wave (XSW) technique in the case of imperfect crystals meets serious theoretical and interpretational problems. However, well-known advantages of the XSW technique make it especially interesting to the study of the ordering of impurities and of various processes leading to changes in this ordering in various types of imperfect crystals. In this work we try to answer the question how imperfection of a crystal may influence the changes in fluorescence yield during the XSW measurement. Two types of imperfect crystals are studied: $\mathrm{Si}(111)$ implanted with high energetic $\mathrm{Bi}^{+}$ions, and $\mathrm{Zn}_{1-x} \mathrm{Co}_{x}$ Se single crystal with natural (111) face. The discussion of obtained results shows that general features of the X-ray standing wave field are conserved despite the considerable imperfections of the crystals. The results seem to support the applicability of the XSW technique to the study of imperfect materials, although some further theoretical effort would be required.
\end{abstract}

PACS numbers: 61.10.-i

\section{Introduction}

In the recent years the $\mathrm{X}$-ray standing wave technique has been extensively applied as one of the most effective methods for non-destructive analysis of the distribution and ordering of impurity atoms in a near-perfect crystal lattices [1]. The number of papers devoted to the quasi-perfect crystal XSW problem has exceeded probably a hundred. However, in case of materials where the approximation of quasi-perfect lattice seems to be not quite satisfied the method meets serious interpretational problems. Well-known advantages of the XSW technique make it especially interesting to the study of the impurity ordering and related processes in such materials. Solving these problems would not only extend the applications of the X-ray standing wave technique. It would also help to answer the basic questions concerning the limitations and sensitivity of the method, also in classical cases of quasi-perfect crystals.

The main aim of this work is therefore to study how the XSW technique applies in two common cases of imperfect crystals when the approximation of 
a near-perfect crystal lattice is not well-satisfied. The first one is an example of XSW measurements of Si wafers implanted with heavy ions, and after that locally annealed with laser. The measured signal from such materials comes from near-surface layers, strongly disturbed because of large radiation damage, but the deeper parts of the crystal remain unchanged, contributing thus in an almost undisturbed X-ray standing wave field.

The other case is the natural crystal face doped during its growth, where the amount of defects can be considerably large. Here the density of defects, comparing to the first case, can be treated as approximately isotropic bulk property of the material and thus influencing the standing wave field in other way.

As an example of the first case the studies are considered, which were partly published earlier [2], of $\mathrm{Si}(111)$ crystals implanted with $\mathrm{Bi}^{+}$ions of $100 \mathrm{keV}$. An evidence of the distribution of the implanted ions and imperfections not only in-depth but also along the surface of the crystal is shown. Basing on the simple computational scheme described fully elsewhere [2], it is possible to estimate lattice constants and ion positions in some types of implanted areas in the crystal.

The example of the second case is $\mathrm{ZnSe}(111)$ natural face crystal doped with $5 \%$ Co atoms during its growth. In order to correlate qualitatively the information about defects and atom positions an attempt of measurements both of the XSW signal and the X-ray reflection topography was done. The same device for both types of measurements was applied. Such an arrangement makes it possible to obtain the XSW spectra exactly from the crystal areas of particular defect pattern.

\section{Experimental setup}

All measurements were done with the XSW device fully constructed at the Institute of Physics, Polish Academy of Sciences. The radiation applied for the measurements was Mo $K_{\alpha 1}$. The construction of the device was based on the rebuilt classical double-crystal $\mathrm{X}$-ray device with conventional $\mathrm{X}$-ray tube as a radiation source. Its general view is presented in Fig. 1. The mechanical part of the sample crystal movement is equipped with step motor for fine rotation necessary in XSW measurements; an analog motor maintains the crystal movement parallel to its surface enabling thus some other kinds of measurements, e.g. recording of the $\mathrm{X}$-ray topograms applied in this work. The detailed description of the spectrometer was published elsewhere $[2,3]$.

\section{Sample preparation and tests}

The $\langle 111\rangle$ oriented Si wafers were implanted with the $\mathrm{Bi}^{+}$ions at the energy of $100 \mathrm{keV}$ and dose of $1.0 \times 10^{+16}$ ions. $\mathrm{cm}^{-2}$. After implantation the samples were locally annealed by a high-power pulsed laser. TEM investigations carried out with carefully chemically thinned samples showed in unannealed parts of the wafer the diffuse diffraction rings characteristic of an amorphous state. The respective micrographs indicated homogeneity with the contrast typical for such cases. In the annealed part of the crystal the complete recrystallization of the implanted layer 


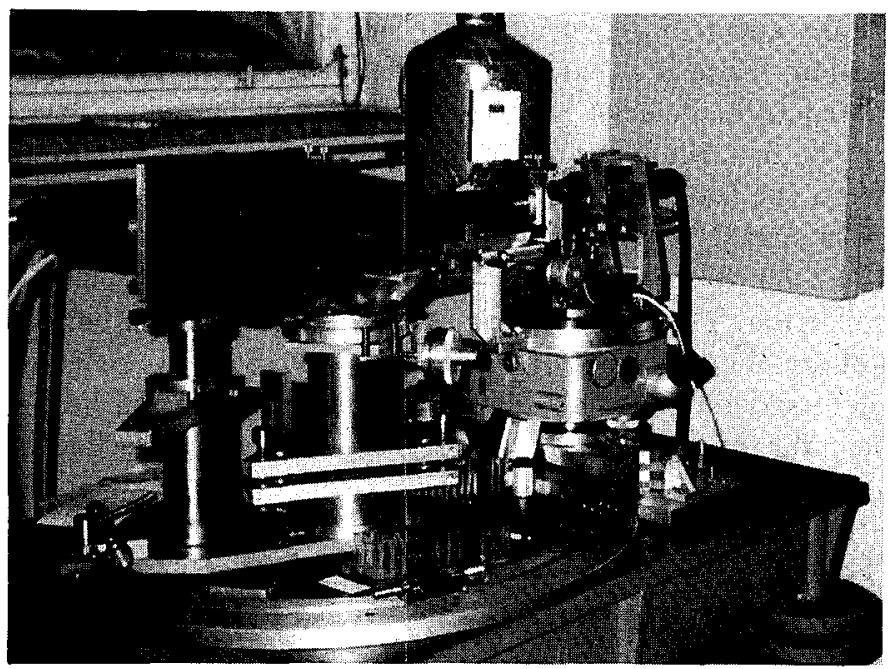

Fig. 1. General view of the XSW spectrometer setup used for the measurements.

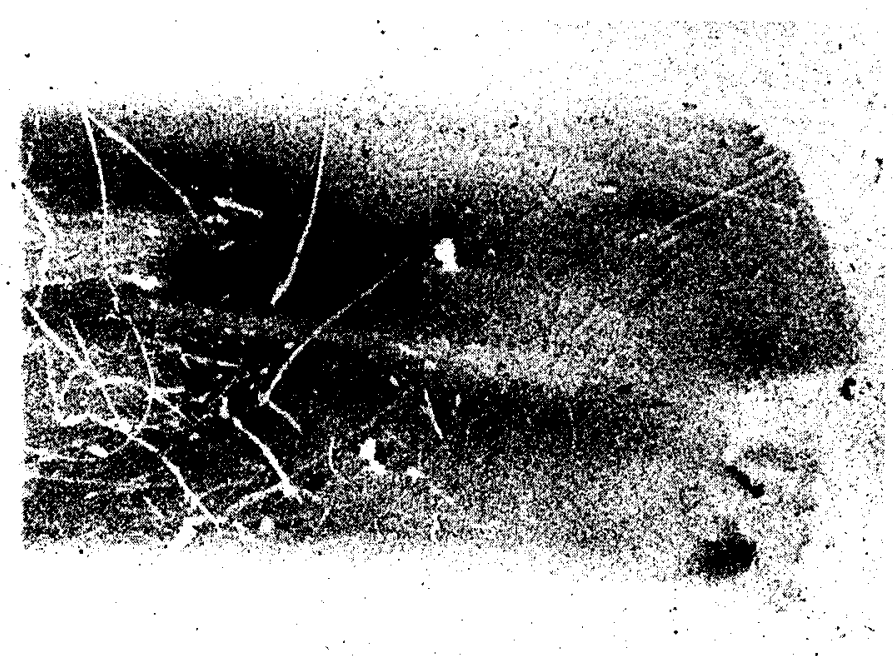

Fig. 2. X-ray reflection topogram of $\mathrm{ZnSe}(\mathrm{Co})(111)$ natural face crystal (magnification $10 \times)$.

and inhomogeneous distribution of extended defects were observed. The recrystallization left a large number of inhomogeneously distributed defects (i.e. microtwins and very small dislocation loops), after [4].

$\mathrm{Zn}_{1-x} \mathrm{Co}_{x}$ Se single crystal with natural (111) face was obtained by chemical vapour transport. The cobalt concentration was $5 \%$. The details of its preparation will be published elsewhere [5]. Double-crystal spectrometry was applied for the 
$\mathrm{X}$-ray topography and rocking curve measurements. The spectrometer was set up in $(+,-)$ position. Since it is extremely difficult to find a $\mathrm{ZnSe}$ reference crystal without any structural defects, we used instead a silicon single crystal of (111) orientation as the first monochromator of the X-ray beam. This monochromator was cut off from the bulk with asymmetry parameter about 0.1 . Such an experimental set was slightly dispersive ( $\mathrm{Si}$ crystal, $a_{0}=5.43095 \AA$ and $\mathrm{ZnSe}(\mathrm{Co})$, $a_{0}=5.66910 \pm 2 \AA$ ). The $\mathrm{X}$-ray radiation from Mo tube was monochromatized using (111) reflection. The halfwidth measured for reference Si perfect crystal was 3.56 seconds of arc. Theoretically the halfwidth of $\mathrm{ZnSe}$ (111) reflection, assuming strictly parallel incident wave, is 7.82 seconds of arc. Measured halfwidths for the investigated crystal ranged from $9.5^{\prime \prime}$ to $16^{\prime \prime}$ depending on the illuminated part of the crystal.

The X-ray double-crystal reflection topograms of $\mathrm{ZnSe}(\mathrm{Co})$ crystal obtained by Mo $K_{\alpha 1}$ (111) reflection are shown in Fig. 2. They reveal the number of extended surface defects (scratches, dislocations, and small cavities).

\section{Experimental results and discussion}

\subsection{Implanted Si crystal}

Figure 3 represents the XSW measurements of the Si(111) wafer implanted with $\mathrm{Bi}$. On the experimental curves the results of the computer analysis of the data are superimposed. A, B, C curves depict the experimental data. A - the rocking curve, B and C - the Bi fluorescence. The figures RA and FA are for the annealed sample; FU is for the unannealed sample. The solid lines on each picture show the results of computer modelling. The angular distributions of fluorescence yield were measured as a sum of integrated fluorescent peaks $\mathrm{Bi} L_{\alpha}+\mathrm{Bi} L_{\beta}$. The shapes of the experimental curves with few pronounced maxima and minima suggest a rather complex situation in the wafer after implantation. In the measured reflectivity one can distinguish four characteristic features.

The first feature with the highest maximum corresponds to the implanted unannealed material. Its lattice parameter is the same as in pure $\operatorname{Si}(111)\left(d_{111}=\right.$ $3.13532 \AA$ ). The second region is pronounced by a shallow minimum. The last two features correspond to the implanted regions after laser annealing.

In order to analyse the results, a computer simulation was applied, based on an assumption that the experimental reflectivity can be synthesized from a few elemental (4 in the discussed case) rocking curves similar to that of pure $\operatorname{Si}(111)$ and shifted against each other. It is equivalent to the model of a crystal with a discrete distribution in lattice constant due to regions with various concentrations of extended defects, and various impurity distribution functions. Next, the fluorescence curve is simulated, based on the parameters defined during the reflectivity simulation. The simulation procedure was described in our previous paper [2]. Comparison of the simulated curves with the experimental in case of the annealed sample shows satisfactory similarity except for the region of the shallow minimum. The experimental reflectivity was constructed with four components. Component 3 is shifted of about 10 seconds in relation to the main peak 1 , and its 

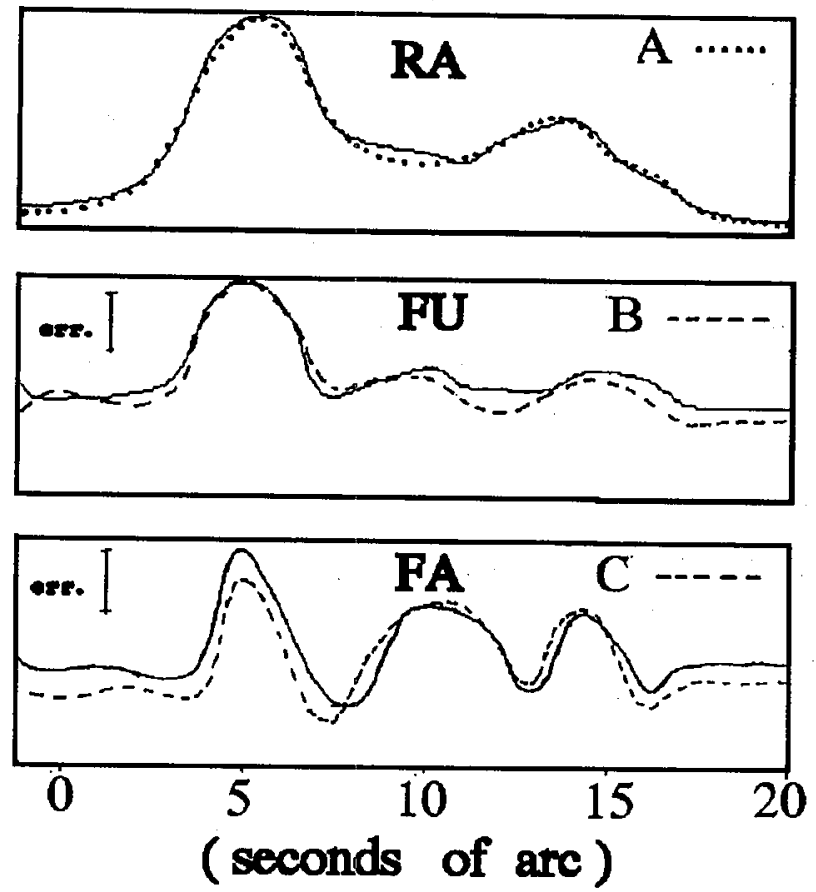

Fig. 3. XSW measurements of the $\mathrm{Si}(111)$ wafer implanted with $\mathrm{Bi}$. A, B, C lines represent the experimental data: (A) - the rocking curve, (B) and (C) - the Bi fluorescence. The figures $\mathrm{RA}$ and FA are for the annealed sample; FU is for the unannealed sample. The solid lines on each picture show the results of computer modelling. See text for details.

intensity is about $40 \%$ of that peak. Component 4 is shifted of about 12.5 seconds in relation to peak 1 , and its intensity is only about $18 \%$ of the main peak. It indicates that the majority of the implanted impurities after annealing occupy crystal areas of two dominating lattice parameters: for the component $3, d_{111}=3.13398$ $\AA$, and for the component $4, d_{111}=3.13365 \AA$. It was not possible using this simple assumption to synthesize the region 2 of the experimental rocking curve with its shallow minimum. In our present opinion it can be due to existence in the implanted crystal of a large amount of relatively small areas with various lattice constants coming from various concentrations of extended defects (e.g. stresses or strains) $[2,6]$.

The results of simulation of the experimental fluorescence curve clearly show that the maximum of the fluorescence main peak 1 is shifted of about $1^{\prime \prime}$ with respect to the corresponding peak of the rocking curve. It suggests that the implanted $\mathrm{Bi}$ atoms, after annealing, are not randomly deposited in the crystal, and there is a preferred position of the impurity atoms with respect to reflecting planes. It was not possible to construct the theoretical fluorescence curve corresponding 
to the experimental result without additional assumption that in the areas of the crystal with various main lattice constants there are various concentrations of $\mathrm{Bi}$ impurity. The simulation indicated also that the concentration of the Bi impurities increases at the surface after the annealing. These conclusions are consistent with the observations of other authors. The disorder of the Bi impurities clearly decreases in the case of areas ascribed to peak 4 . It is more difficult to answer the similar question concerning the areas ascribed to the peak 3 , because it can be highly disturbed by, discussed earlier, large amount of small areas with various lattice constants, due to the different concentration and ordering of the impurity. In this area a lot of $\mathrm{Bi}$ atoms were placed in the substitutional position.

An attempt to evaluate the preferred positions of the $\mathrm{Bi}$ impurities after annealing was done. In the regions 1 and 4 the preferred positions were estimated to be less than $0.2 d_{111}$ from the reflecting planes. It is consistent with the observations of other authors that Bi atoms implanted in Si crystal tend to occupy substitutional positions even when the solid solubility limit is exceeded [7]. Similar evaluations for regions 2 and 3 lead to the conclusion that coherent phase is equal or less to 0.5 in these regions, and evaluation of the preferred positions is practically impossible.

\subsection{ZnSe crystal}

In the both parts of Fig. 4 the results of the XSW measurements of $\mathrm{ZnSe}(\mathrm{Co})$ crystal are shown. The rocking curve is depicted with circles, the thick solid line is for fluorescence yield of $\mathrm{Zn}$ atoms. The thin line in the part (a) of the figure represents the Se fluorescence yield; the thin line in part (b) shows the fluorescence yield of the Co impurity. In all cases the fluorescence was measured as integrated intensity of the appropriate $K_{\alpha}+K_{\beta}$ lines. The experimental rocking curve is much broader than the calculated one and shows some irregularities on its both shoulders. Although we could measure narrower and more smooth rocking curves from other areas of the crystal, this one shown here is very representative. Both from the topograms and from the shape and halfwidth of the rocking curve it is clear that the investigated crystal cannot be considered as a perfect one. Nevertheless, it is evident that the typical effect found in bulk perfect crystals of GaAs type of XSW fluorescence decreasing near the middle of the rocking curve is clearly visible [8]. From our data one can see that the minima of the fluorescent curves for Se and $\mathrm{Zn}$ are shifted one another of about 1-2 angular seconds. The shape of the curves differs also slightly especially at the higher angles. Possible explanation for these differences is that in case of reflection (111) in non-centrosymmetric crystals of GaAs type, the two kinds of atoms are separated on other atomic layers. This causes that the X-ray standing wave field pattern differs for both kinds of atoms. This difference is usually expected to be lowered by self-absorption effect [8]. Unfortunately, in case of the Si:Li detector applied in our XSW experiment it was not possible to change its angle with respect to the sample surface, which is common procedure to change the self-absorption conditions. The statistics of Co fluorescence measurements was poor. Therefore, we can notice only that its general form is similar to the two previous with characteristic minimum in the region of the rocking curve maximum. From this one can conclude that the majority of the Co 

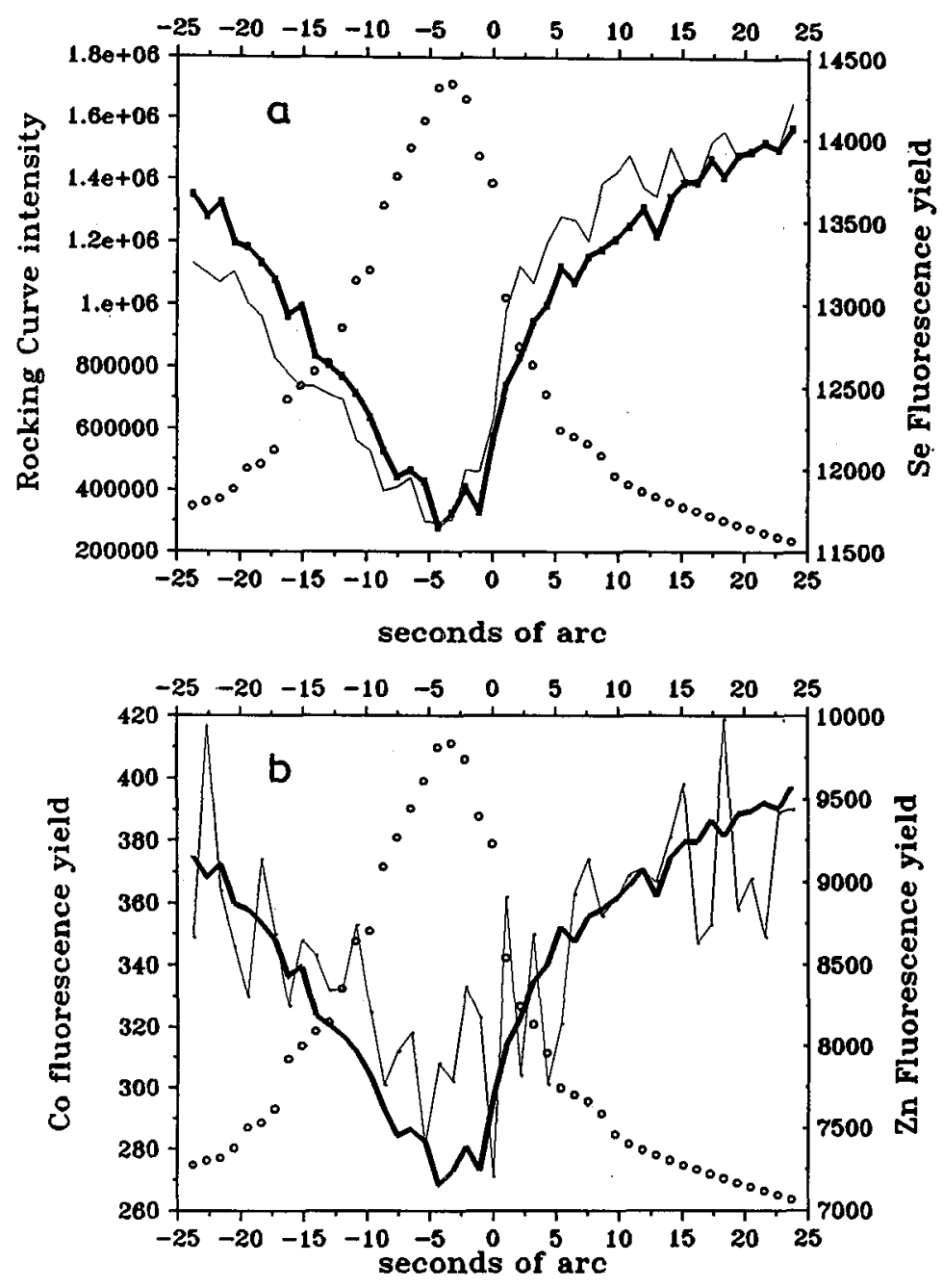

Fig. 4. XSW experimental data for $\mathrm{ZnSe}(\mathrm{Co})$. Circles - rocking curve, solid lines fluorescence yields, thick $-\mathrm{Zn}$, thin $(\mathrm{A})-\mathrm{Se}$, thin $(B)-$ Co.

atoms are in substitutional positions. It is expected that Co substitutes zinc atoms rather than selenium, but we are unable to prove this from our measurements.

\section{Conclusions}

We tried to answer the question how imperfection of crystal surface may influence the changes in fluorescence yield when we scan the investigated crystal across the angular position corresponding to the Bragg one. Various common types of imperfect crystals were represented by $\mathrm{Si}(111)$ implanted with high energetic $\mathrm{Bi}^{+}$ions. $\mathrm{Zn}_{1-x} \mathrm{Co}_{x} \mathrm{Se}$ single crystal with natural (111) face was chosen as an 
example of natural face crystal doped with other impurity, here cobalt.

Despite the complexity of parameters characterizing the investigated imperfect materials, the XSW measurements show that the X-ray standing wave field is created in such materials and investigation with this technique can bring a lot of interpretable features.

In case of the implanted sample the studies allowed to demonstrate that the concentration of the Bi impurities increases after annealing at the surface, and their ordering increases significantly. The samples implanted with heavy ions can be composed of some types of areas of various lattice constants, caused by different concentrations of impurity atoms, their positions and disorder. The distribution of the defects and lattice constants not only in-depth of the crystal but also along its surface should be taken into account. Comparison of the modelled reflectivity curve with the experiment shows good agreement except for the region of shallow minimum, which can be the result of existence of a lot of small areas of intermediate lattice constants not well-maintained within the model. In the implanted and annealed crystal the synthesized fluorescence, although it does not so well agree with the experimental one, indicates that most of the impurities occupy areas of two dominating lattice constants, and their positions are highly coherent. In the samples before annealing impurity positions of higher disorder are observed. The presented study showed both increase in ordering of the impurities after laser annealing, as well as relevance of the XSW method for the non-destructive monitoring of impurity atoms distribution changes due to laser annealing.

In case of natural faced $\mathrm{ZnSe}(\mathrm{Co})$ crystal it was demonstrated that the general features found in XSW measurements of near-perfect crystals of the similar type are present despite of the documented imperfection of the studied sample. The extended defects in the small angular grain boundary and dislocations have not so large effect on the character of the angular fluorescence yield distribution, but some perturbations of its form can be found.

\section{Acknowledgments}

The authors wish to thank Mr. Jerzy Dąbrowski for his technical assistance during XSW measurements.

This work was partially sponsored by the State Committee for Scientific Research (No. 935/2/91).

\section{References}

[1] B.W. Batterman, Phys. Rev. A 133, 759 (1964).

[2] J. Auleytner, P. Dłużewski, J. Pełka, in: Proc. 1st Europ. Conf. Radiat. Effects Dev. Syst. RADECS 91, La Grande Motte (France), Vol. 15, Ed. J.-P. Charles, L. Adams, A. Holmes-Siedle, IEEE, 1991, p. 544.

[3] J. Auleytner, J. Majewski, Z. Furmanik, Z. Gołacki, Cryst. Res. Technol. 25, 971 (1990).

[4] J. Morawiec, J. Auleytner, D. Wieluniska, in: Proc. 7th Int. School, Defects in Crystals, Szczyrk (Poland), 1985, Ed. E. Mizera, 1985, p. 462. 
[5] J. Auleytner, Z. Golacki, et al., to be published.

[6] J. Auleytner, J. Majewski, Surf. Sci. 231, 227 (1990).

[7] N. Hertel, G. Materlik, J. Zegenhagen, Z. Phys. B 58, 199 (1985).

[8] J.R. Patel, J.A. Golovchenko, Phys. Rev. Lett. 50, 1858 (1983). 